\title{
Assessment of Knowledge and Practices of myocardial Infarction' Patients After Primary Percutaneous Coronary Intervention at Outpatient Clinic in Heart Hospital Assiut University
}

\author{
Safaa Omran Kamal ${ }^{1}$, Hoda Diab Fahmy ${ }^{2,}$ Hosam Hasan Ali ${ }^{3}$, Soad AbdElhamed Sharkawy ${ }^{4}$, Safaa Rashad \\ Mahmoud 5 . \\ 1. Professional nurse Heart Hospital, Egypt. \\ 2. Professor of Community Health Nursing Faculty of Nursing, Egypt. \\ 3. Professor of Cardiology Faculty of Medicine, Egypt. \\ 4. Assistant Professor of Community Health Nursing Faculty of Nursing, Egypt. \\ 5. Assistant Professor of Community Health Nursing Faculty of Nursing, Assiut University Egypt.
}

\begin{abstract}
Primary percutaneous coronary intervention (PPCI) is an emergent percutaneous catheter intervention in the setting of ST elevation myocardial infarction, it is the preferred reperfusion strategy. Aim of the study: Assessment of knowledge and practices of myocardial infarction patients after (PPCI). Study design: A descriptive cross- sectional study research design has been utilized. Setting: the study was conducted at outpatient clinic Cardiovascular, Heart Hospital Assiut University. Subjects: A convenience sample of 150 patients was included in the study. Tools: A structured interview questionnaire including three parts included personal data, patients' knowledge about myocardial infarction, (PPCI)and reported practices Results: It was found that 43,3\% of patients their age were younger than 50 year and $77.0 \%$ of them were males also it was found that $58.0 \%$ of patients had poor knowledge about myocardial infarction and (PPCI). More than three quarters of patients always take food rich in fat. $60.7 \%$ of them never practice any kind of exercise, $83.3 \%$ of them were smokers. Conclusion: There was a great lacking of the necessary basic knowledge and practice of studied patients about myocardial infarction after (PPCI). Recommendations: Health education program is needed for patients with myocardial infarction to improve their knowledge and practice regarding their disease.
\end{abstract}

\section{Keywords: Myocardial infarction, primary percutaneous Coronary Intervention. Knowledge \& Practice.}

\section{Introduction}

Myocardial infarction (MI) or heart attack occurs if the flow of oxygen-rich blood is cut off to a section of heart muscle. This can happen if an area of plaque in a coronary artery ruptures. Blood cell fragments called platelets stick to the site of the injury and clump together to form blood clots. If a clot becomes large enough, it can mostly or completely block blood flow through a coronary artery. (Gary \& Gibbons, 2015).

ST-segment elevation myocardial infarction (STEMI) is the term used by cardiologists to describe the classic heart attack. It is a type of myocardial infarction in which a part of the heart muscle (myocardium) has died due to the obstruction of blood supply to the area. The main symptoms of MI include chest pain, which travels from left arm to neck, shortness of breath, sweating, nausea, vomiting, abnormal heart beating, anxiety, fatigue, weakness, stress, depression. (Maziar \& Eric., 2016).

An MI requires immediate medical attention. Treatment attempts to save as much viable heart muscle as possible and prevent further complications, hence the phrase "time is heart muscle". Aspirin and nitroglycerin may be administered. Nitroglycerin administered under the tongue or intravenously to improve the blood supply to the heart. Morphine also may be used if nitroglycerin is not effective. Analgesics such as nitrous oxide are of unknown benefit (Amsterdam. et al.,2014).

According to the latest World Health Organization data published in 2017 coronary heart disease deaths in Egypt reached 126,312 or $24.58 \%$ of total deaths. The age adjusted Death Rate is 216.82 per 100,000 of population. (World health rankings 2017).

Primary percutaneous coronary intervention (PPCI): an emergent percutaneous catheter intervention in the setting of STEMI, without previous fibrinolytic treatment, and preferred reperfusion strategy in patients with STEMI, PPCI is now the preferred treatment of choice for patients with STEMI and now makes up more than $97 \%$ of reperfusion treatment for patients with a final diagnosis of STEMI. Coronary angioplasty is widely practiced and has a number of risks, however, major procedural complications are bleeding, infection, allergic, death, stroke, heart attack are uncommon (Harold, et al., 2013).

Surviving a heart attack is often a life-changing event. In addition to recovering from any procedures that were performed to stop and treat the heart attack,

Vol , (7) No, (17) June, 2019 
most patients will need extensive lifestyle changes. These lifestyle changes are designed to target risk factors for heart disease and stop or slow the progress of disease. Making lifestyle changes is not easy, doing so after a heart attack is an important part of looking toward the future. (Wright et al., 2015). Worldwide, about 15.9 million myocardial infarctions occurred in 2015. More than 3 million people had an ST elevation MI and more than 4 million had an NSTEMI. non-ST elevation myocardial infarction (Global Burden of Disease 2016).

Nurses must enhance patient self-care by assessing the health needs of their patients and providing appropriate discharge planning. The learning needs of cardiac patients include recognizing and managing symptoms, medication management and lifestyle factors. Nurses represent a large proportion of health care community. They play a vital role in treatment as they are close to the patients and their families during all the process of disease. (Goel, et al., 2011).

\section{Significance of the study}

Acute myocardial infarction (MI) is associated with a $30 \%$ mortality rate; about $50 \%$ of the deaths occur prior to arrival at the Canadian vigour hospital, University of Alberta, Canda. An additional 5-10\% of survivors die within the first year after their myocardial infarction. Approximately half of all patients with an MI are rehospitalized within 1 year of their index event. Successful early reperfusion depends on patient arrival to fibrinolysis infusion within 30 minutes or patient arrival to percutaneous coronary intervention [PCI] within 90 minutes (Alherbish, et al., 2013).

In Assiut University Hospital, department of cardiology, The incidence of hospital admission for Acute myocardial infarction (MI) from August 2015 to August 2016 about 800 cases 300 of them underwent primary percutaneous coronary intervention (PPCI), ( 25 to 30 patient by month). They discharged after 2-5 days from coronary care unit (Coronary care unit, Assiut University hospital 2016).

\section{Aim of the study}

To assess patients's knowledge and practice about myocardial infarction after primary percutaneous coronary intervention (PPCI).

\section{Research questions}

1- What are the levels of patients' knowledge about myocardial infarction after primary percutaneous coronary intervention?

2- What are the levels of patients' reported practice about myocardial infarction after primary percutaneous coronary intervention?

\section{Subjects \& Methods \\ Study design}

- A descriptive cross- sectional study research design has been utilized in the current study.

Setting

- The study was conducted in Cardiovascular Outpatient Clinics at Heart Hospital Assiut University.

\section{Sampling:}

- A convenient sample of 150 patients was included in the study.

\section{Study tool}

A structured interview form was conducted after reviewing the relevant literature to elicit information. It was structured into three parts.

\section{The first part}

It included socio demographic characteristics such as patient's name, age, sex, level of education, marital status, occupation, residence, address, date of operation.

\section{The second Part}

It included (19) questions regarding patients' knowledge about myocardial infarction, percutaneous coronary intervention and lifestyle pattern. (14) Questions about reported practice as following.

- Patients' knowledge about the myocardial infarction:

It included (6) questions as (Definition, symptoms, main cause, risk factors, actions that must be taken if there is chest pain, and types of treatment).

- Patients' knowledge about primary percutaneous coronary intervention (PPCI):

It included (8) questions as (Definition, appropriate reperfusion strategy, advantage, medication must be taken after PPCI, complications and how to reduce these complications).

- Patients' knowledge about lifestyle pattern:

It included (5) questions as (smoking hazard, benefits of smoking cessation , return to work, return of sexual activity and precautions when resumption of sexual activity)

- Reported practices questionnaire : It included (14) questions regarding patients' assessment of lifestyle pattern (is the patient take in his diet foods that contain fiber, fruits and vegetables, food rich in fat, take a lot of sugars or sugar products, a lot of salt, drink tea or coffee. Practices any kind of exercise, how many hours he spend in his exercise, practice exercises under the supervision of a physician, types of exercise and benefit of exercise. Also is he smoker now and if he intend to quit smoking).

Scoring system: The total score of knowledge were 19 grads it developed as one grad for each correct answer and zero for each incorrect answer. For each area of knowledge, the scores of the items were 
summed-up and the total divided by the number of the items, giving a mean score for the part. These scores were converted into a percent score as the following. Poor $<50 \%$, Fair $50 \%<70 \%$, Good $>70 \%$. Scoring system for reported practice, the responses always, sometime and never were respectively scored 3,2 and 1 . These scores were converted into a percent score as the following. Inadequate $<50 \%$ and adequate $<50 \%$.

Third Part:

Patient health assessment which include:

A- Medical history

(Risk factors - family history - Past complain -

Medication - drugs and surgical intervention

presenting symptoms of coronary artery disease).

B- Physical examination

- Inspection of Face- Jugular veins- Thorax- Nail beds- Abdomen- Lower extremities.

- Palpation (Pulse- Capillary refill- Lower limb edema- Blood Pressure)

- Psychological problems.

- Validity of the tool

The tool were revised by a jury of five experts nursing and medical staff whom reviewed the instrument for clarity, relevance, comprehensiveness, understanding and applicability. Recommended modifications were fulfilled.

- Reliability of the tool

Reliability of the tool was assessed using alpha test to test the internal consistency knowledge $=0.788$

Methodology

Preparatory phase and administrative design

- An official approval was obtained from the Dean of Faculty of Nursing, Assiut University to the head of the cardiology Hospital at Assiut University, to carry out this study and explained the purpose of the study, and asking for permission to conduct it.

\section{Pilot Study}

After developing the necessary tool of the study. A Pilot study included $10 \%$ of patients which was carried out to ensure the clarity and applicability of the developed tool, and to estimate the time required to fill the questionnaire. Based on the results of the pilot study, the necessary modifications were done such as (types of exercise, pattern of food) Patients included in the pilot study were excluded from the study sample.

\section{Ethical consideration}

Research proposal was approved from Ethical committee in the Faculty of Nursing at Assiut University. There is no risk for study subject during application of the research. The study will follow common ethical principles in clinical research. Confidentiality and anonymity will be assured. Study subject have the right to refuse to participate and or withdraw from the study without rational at any time. Study subject privacy will be considered during collection of data and agree to participant in this study (oral agreement).

\section{Statistical analysis}

Date entry and data analysis were done using SPSS version 19 (Statistical Package for Social Science). Data were presented as number, percentage, mean, standard deviation. Chi-square test and Fisher Exact test were used to compare qualitative variables. Pvalue considered statistically significant when $\mathrm{P}<$ 0.05 .

\section{Field of work}

The researchers started to collect data from the first of January 2018 until the end of March 2018. The researchers met the patients in cardiovascular outpatient clinics at Heart Hospital, Assiut University, they introduced themselves to the participants to establish the necessary rapport and gain their attention and interest, explain the purpose of the study. Confidentiality and anonymity would be assured. They collect data during interview, each interview take 30 minutes, the data were collected in 12 weeks. 


\section{Results}

Table (1): Distribution of studied patients regarding to their socio demographic characteristics at Assiut University Heart Hospital in (2017) no=(150).

\begin{tabular}{|l|c|c|}
\hline \multicolumn{1}{|c|}{ personal characteristics } & No. & \% \\
\hline Age:(years) & \multicolumn{2}{|c|}{4} \\
\hline$<50$ & \multicolumn{2}{|c|}{$49.86 \pm 9.16(35.0-67.0)$} \\
\hline$\geq 50$ & \multicolumn{2}{|c|}{56.7} \\
\hline Mean \pm SD (Range) & 116 & 77.3 \\
\hline Sex: & 34 & 22.7 \\
\hline Male & & \\
\hline Female & 75 & 50.0 \\
\hline Residence: & 75 & 50.0 \\
\hline Urban & & 10.0 \\
\hline Rural & 15 & 67.3 \\
\hline Marital status: & 101 & 22.7 \\
\hline Single & 34 & \\
\hline Married & & \\
\hline Widow & 32 & 21.3 \\
\hline Level of education: & 30 & 20.0 \\
\hline Illiterate & 20 & 13.3 \\
\hline Read and write & 41 & 27.3 \\
\hline Basic education & 27 & 18.0 \\
\hline Secondary education & & \\
\hline University education & & \\
\hline
\end{tabular}

Table (2): Distribution of studied patients regarding to their medical history at Assiut University Heart Hospital (2017). $n=(150)$.

\begin{tabular}{|l|c|c|}
\hline \multicolumn{1}{|c|}{ Item } & \multicolumn{2}{c|}{ (n= 150) } \\
\cline { 2 - 3 } & No. & \\
\hline Risk factors:\# & & \\
\hline Hypertension (high blood pressure) & 51 & 34.0 \\
\hline Unhealthy diet & 95 & 63.3 \\
\hline Diabetes Mellitus & 38 & 25.3 \\
\hline Physical inactivity & 53 & 35.3 \\
\hline Tobacco use & 125 & 83.3 \\
\hline Family history: & & \\
\hline Hadn't family history & 53 & 35.3 \\
\hline Cardiovascular disease before the age of 55 years & 80 & 53.3 \\
\hline Hypertension & 17 & 11.3 \\
\hline Past complain: & & \\
\hline Hadn't past complain & 145 & 96.7 \\
\hline Heart failure & 4 & 2.7 \\
\hline Coronary artery disease & 1 & 0.7 \\
\hline Medication: & & \\
\hline No medication & 115 & 76.7 \\
\hline Nitrates +Aspirin & 35 & 23.3 \\
\hline
\end{tabular}

\# More than one answer 
Table (3): Distribution of studied patients regarding to their knowledge about acute myocardial infarction at Assiut University, Heart Hospital (2017). $n=(150)$.

\begin{tabular}{|c|c|c|}
\hline Items & No. & $\%$ \\
\hline \multicolumn{3}{|l|}{ Definition of acute myocardial infarction: } \\
\hline Correct answer & 24 & 16.0 \\
\hline \multicolumn{3}{|l|}{ Symptoms of myocardial infarction \# } \\
\hline Pressure or tightness in the chest & 100 & 66.7 \\
\hline Pain in the chest, back, jaw, and other areas of the upper body & 90 & 60 \\
\hline Shortness of breath & 61 & 40.7 \\
\hline Sweating & 77 & 51.3 \\
\hline Nausea & 48 & 32.0 \\
\hline Vomiting & 57 & 38.0 \\
\hline A cough & 36 & 24.0 \\
\hline Dizziness & 61 & 40.7 \\
\hline Fast heart rate & 12 & 8.0 \\
\hline \multicolumn{3}{|l|}{ The main cause of myocardial infarction } \\
\hline Correct answer & 11 & 7.3 \\
\hline \multicolumn{3}{|l|}{ Risk factors for myocardial infarction\# } \\
\hline Don't know & 26 & 17.3 \\
\hline High blood pressure & 16 & 10.7 \\
\hline High cholesterol levels & 56 & 37.3 \\
\hline Diabetes and high blood sugar levels & 33 & 22.0 \\
\hline Obesity & 47 & 31.3 \\
\hline Smoking & 111 & 74.0 \\
\hline Family history & 43 & 28.7 \\
\hline Age & 58 & 38.7 \\
\hline Stress & 66 & 44.0 \\
\hline Lack of exercise & 41 & 27.3 \\
\hline \multicolumn{3}{|l|}{ Ways to treat the disease \# } \\
\hline Don't know & 69 & 46.0 \\
\hline Nutritional therapy & 34 & 22.7 \\
\hline Complete rest & 51 & 34.0 \\
\hline Medical therapy & 54 & 36.0 \\
\hline Reduce the intake of salts & 50 & 33.3 \\
\hline Minimizing fluid & 34 & 22.7 \\
\hline Surgical intervention & 33 & 22.0 \\
\hline \multicolumn{3}{|l|}{ Actions must be taken if there is chest pain\# } \\
\hline Take analgesic & 72 & 48.0 \\
\hline Go to hospital & 69 & 46.0 \\
\hline Go to private cardiologic clinic & 41 & 27.3 \\
\hline
\end{tabular}

\# More than one answer

Table (4): Distribution of studied patients regarding to their knowledge about Primary percutaneous coronary intervention (PPCI) at Assiut University Heart Hospital (2017). ( $n=150)$.

\begin{tabular}{|c|c|c|}
\hline Items & No & $\%$ \\
\hline \multicolumn{3}{|c|}{ Definition of Primary percutaneous coronary intervention (PPCI): } \\
\hline Correct & 19 & 12.7 \\
\hline \multicolumn{3}{|l|}{ The appropriate reperfusion strategy } \\
\hline Incorrect & 150 & 100.0 \\
\hline \multicolumn{3}{|l|}{ complications after (PPCI) } \\
\hline Incorrect & 150 & 100.0 \\
\hline Type of complications after (PPCI) & & \\
\hline
\end{tabular}

Vol , (7) No , (17) June, 2019 


\begin{tabular}{|l|c|c|}
\hline \multicolumn{1}{|c|}{ Items } & No & $\%$ \\
\hline Don't know & 150 & 100.0 \\
\hline Actions can be taken to reduce complications\# & & \\
\hline Don't know & 150 & 100.0 \\
\hline Medication must be taken after PPCI:\# & & \\
\hline Don't know & 150 & 100.0 \\
\hline Duration of using medication & & \\
\hline Don't know & 150 & 100.0 \\
\hline
\end{tabular}

Table (5): Distribution of studied patients regarding to their reported practices at Assiut University Hospital (2017). $(n=150)$.

\begin{tabular}{|l|c|c|}
\hline \multicolumn{1}{|c|}{ Item of reported practices } & No. & \% \\
\hline $\begin{array}{l}\text { Taking diet foods that contain fiber, fruits and vegetables } \\
\text { Always }\end{array}$ & 44 & 29.3 \\
\hline Sometime & 106 & 70.7 \\
\hline Never & 0 & 0.0 \\
\hline Taking diet food rich in fat and grease always & 116 & 77.3 \\
\hline Sometime & 34 & 22.7 \\
\hline Never & 0 & 0.0 \\
\hline $\begin{array}{l}\text { Taking lot, of sugars or and sugar products. } \\
\text { Always }\end{array}$ & 90 & 60.0 \\
\hline Sometime & 60 & 40.0 \\
\hline Never & 0 & 0.0 \\
\hline $\begin{array}{l}\text { Aaking a lot of salt in your food } \\
\text { Always }\end{array}$ & 140 & 93.3 \\
\hline Sometime & 10 & 6.7 \\
\hline Never & 0 & 0.0 \\
\hline $\begin{array}{l}\text { Drinking tea } \\
\text { Always }\end{array}$ & 121 & 80.7 \\
\hline Sometime & 29 & 19.3 \\
\hline Never & 0 & 0.0 \\
\hline $\begin{array}{l}\text { Drinking coffee } \\
\text { Always }\end{array}$ & & \\
\hline Sometime & 80 & 53.3 \\
\hline Never & 29 & 19.3 \\
\hline $\begin{array}{l}\text { Practice any kind of exercise. } \\
\text { Always }\end{array}$ & 41 & 27.3 \\
\hline Sometime & 26 & 17.3 \\
\hline Never & 33 & 22 \\
\hline $\begin{array}{l}\text { Number of smokers } \\
\text { Yes }\end{array}$ & 91 & 60.7 \\
\hline No & 125 & $83.3 \%$ \\
\hline $\begin{array}{l}\text { Number who intend to stop smoking } \\
\text { Yes }\end{array}$ & 25 & 16.7 \\
\hline No & 110 & 88.0 \\
\hline & 15 & 12.0 \\
\hline
\end{tabular}


Table (6): Relationship between total scoreof knowledge and personal data at Assiut University Hospital (2017). $(\mathbf{n}=150)$.

\begin{tabular}{|c|c|c|}
\hline \multirow{2}{*}{ Item } & Knowledge score & \multirow{2}{*}{ P-value } \\
\hline & Mean \pm SD & \\
\hline \multicolumn{2}{|l|}{ Age:(years) } & \multirow{3}{*}{0.327} \\
\hline$<50$ & $18.32 \pm 3.93$ & \\
\hline$\geq 50$ & $17.72 \pm 3.58$ & \\
\hline \multicolumn{2}{|l|}{ Sex } & \multirow{3}{*}{$0.027 *$} \\
\hline Male & $18.34 \pm 3.53$ & \\
\hline Female & $16.74 \pm 4.17$ & \\
\hline \multicolumn{2}{|l|}{ Residence: } & \multirow{3}{*}{$0.033^{*}$} \\
\hline Urban & $18.63 \pm 3.76$ & \\
\hline Rural & $17.33 \pm 3.62$ & \\
\hline \multicolumn{2}{|l|}{ Marital status } & \multirow{4}{*}{$0.003^{*}$} \\
\hline Single & $14.93 \pm 4.79$ & \\
\hline Married & $18.46 \pm 3.27$ & \\
\hline Widow & $17.91 \pm 3.99$ & \\
\hline \multicolumn{2}{|l|}{ Level of education } & \multirow{6}{*}{$0.017^{*}$} \\
\hline High education & $18.85 \pm 4.92$ & \\
\hline Secondary education & $19.22 \pm 3.07$ & \\
\hline Basic education & $17.05 \pm 3.46$ & \\
\hline Read and write & $17.57 \pm 3.55$ & \\
\hline Illiterate & $16.63 \pm 3.18$ & \\
\hline \multicolumn{2}{|l|}{ Occupation } & \multirow{7}{*}{$0.001 *$} \\
\hline Employee & $20.07 \pm 2.56$ & \\
\hline Farmer & $17.38 \pm 4.50$ & \\
\hline Housewife & $15.72 \pm 3.03$ & \\
\hline Skilled & $18.28 \pm 3.90$ & \\
\hline Unskilled & $18.08 \pm 2.07$ & \\
\hline Free business & $17.68 \pm 4.18$ & \\
\hline
\end{tabular}

Table (7): Total score of reported practice at Assiut University Hospital (2017). ( $(n=150)$

\begin{tabular}{|l|c|c|}
\hline \multicolumn{1}{|c|}{ Item } & No. (150) & \% \\
\hline Inadequate & 133 & 88.7 \\
\hline Adequate & 17 & 11.3 \\
\hline
\end{tabular}

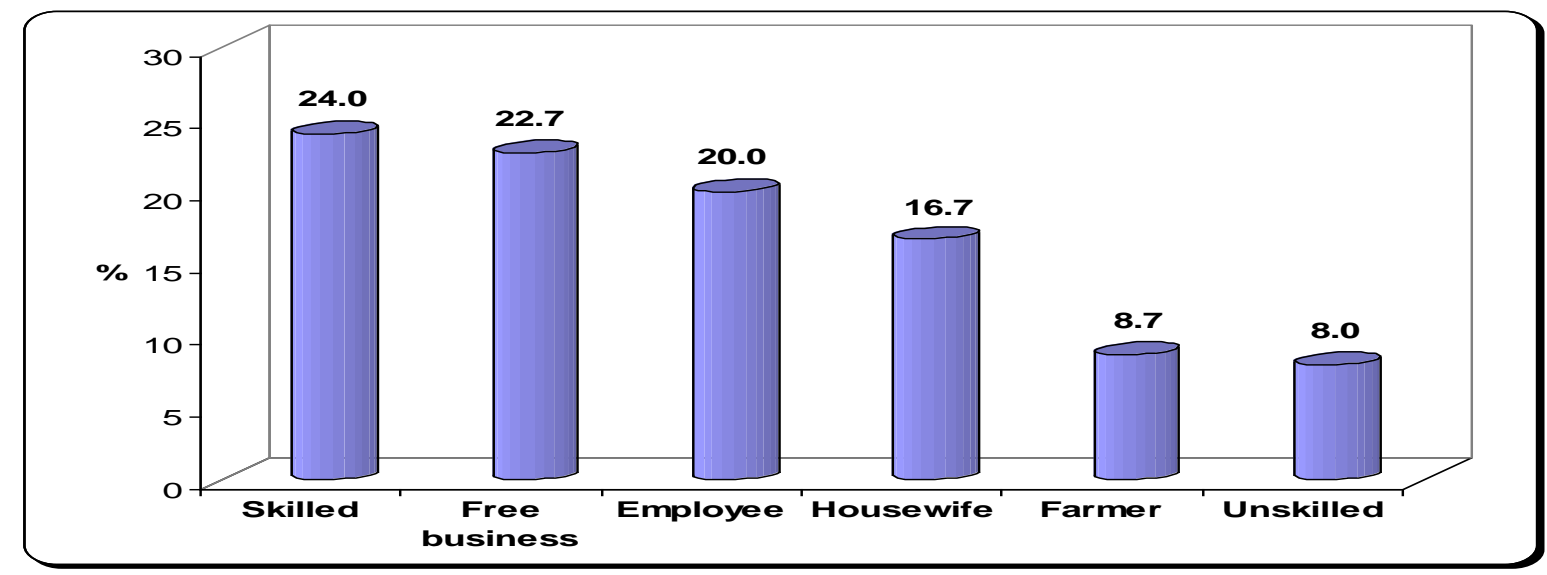

Figure (1): Distribution of the studied patients according their occupation (2017). 


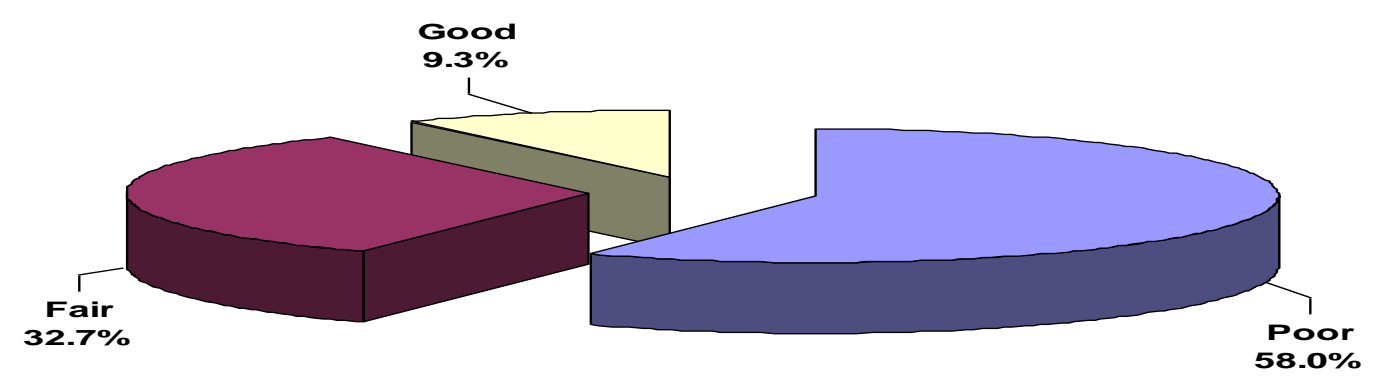

\section{Figure (2): Total score of patients's knowledge about myocardial infarction and primary percutaneous intervention.}

Table (1): Illustrates the distribution of the studied patients regarding their personal characteristics. It was founded that, More than two fifths $(43.3 \%)$ of patients their age younger than 50 years with mean \pm SD $49.86 \pm 9.16$. More than two thirds $(67.3 \%)$ of them were married and $27.3 \%$ of them had secondary education.

Table (2): Shows the distribution of studied patients regarding medical history. It was found that the majority $(83.3 \%)$ of patients had risk factors of smoking. Slightly more than one quarter $(25.3 \%)$ had risk factors of diabetes mellitus. Slightly more than half of them $(50.3 \%)$ had family history of coronary artery disease before the age 55 years. The vast majority $(96.7 \%)$ of patients hadn't past complain.

Table (3): Explains the distribution of studied patients's knowledge about myocardial infarction . It was found that $16.0 \%$ of patients know the definition of acute myocardial infarction correctly. $66.7 \%$ of patients said that pressure or tightness in the chest is symptom of myocardial infarction. $40.7 \%$ of patients explain that shortness of breath is the symptom of myocardial infarction. Only $7.3 \%$ from the total patients know the main causes of myocardial infarction. $74.0 \%$ of patients said that smoking is the most risk factors for myocardial infarction. $22.0 \%$ of patients know that diabetes mellitus is the a risk factors for myocardial infarction.

Table (4): Shows the studied Patients's knowledge about Primary percutaneous coronary intervention (PPCI) . It was found that $12.7 \%$ of patients know the definition of Primary percutaneous coronary intervention correctly. Nobody $00.0 \%$ of patients know that primary percutaneous coronary intervention is the appropriate reperfusion therapy.

Table (5): Shows the distribution of the studied patients regarding to their reported practices. It was found that $77.3 \%$ of patients always taking diet food rich in fat and grease, and $93.3 \%$ of patients always taking a lot of salt in their food. $60.7 \%$ of patients never practice any kind of exercise. $83.3 \%$ of patients were smokers .

Table (6): Explain the relationship between knowledge score and personal data. It was found that their is a statistical significant deference between levels of knowledge and personal data.

Table (7): Shows the total score of reported practice. It was found that the majority $88.7 \%$ of patients had Inadequate level of reported practice.

Figure (1): Distribution of the studied patients according their occupation. It was found that nearly $24.0 \%$ had skilled work, $22 \% 7 \%$ had free business. While only $8.7 \%$ and $8.0 \%$ were (farmer, unskilled) respectively .

Figure (2): Shows total score of patients's knowledge regarding myocardial infarction and primary percutaneous intervention it was found that more than half of patients $58.0 \%$ had poor knowledge.

\section{Discussion}

Primary percutaneous coronary intervention targets early intervention, achieving better outcomes for patients suffering from ST elevation myocardial infarction .primary percutaneous coronary intervention and it is the most commonly performed revascularization method among cardiac patients. Primary angioplasty results in better reperfusion and reduces mortality, frequency of re-infarction and stroke when compared with thrombolytic therapy in patients with ST elevation myocardial infarction. (Wijesinghe et al., 2018).

It was observed in the present study that, more than two fifths of patient their age were younger than 50 years with mean $\pm \mathrm{SD}(49.86 \pm 9.16)$. This agree with (Ahmed 2013) who presented in her study about the impact of phase one cardiac rehabilitation on early complication of patients with St segment elevation acute myocardial infarction in coronary 
care unit that the majority of both groups were in age group with mean and standard deviation of age $(55.4+10.5 \& 57.1+10.0)$.

Old age increase risk of damaged and narrowed arteries. Men who are 45 or older and women who are 55 or older are more likely to have a heart attack than are younger men and women (Crawford et al., 2009)

As regarding the patient sex, the finding of the current study revealed that more than three quarter were male, while less than one quarter were female. This result agreed with (Garza et al., 2009) who reported in study about improved Patient survival using a modified resuscitation protocol for out-ofhospital in the emergency medical system in Kansas City, that three fifths of the samples acute myocardial infarction (AMI) were male and $40 \%$ were female. The risk of having a myocardial infarction in women compared to the men of the same age is one fourth this could be explained through the estrogen hormone, This big difference is reduced after reaching the age of menopause, and at the age of 50 the chance of myocardial infarction becomes equal the Incidence in men and women equalized male's. This contradicts this study with (Mehta, et al., 2016) Who reported that after age 65 the increasing incidence of MI at females more than males, In the first scientific statement from the American Heart Association on acute myocardial infarction in women.

Regarding to the marital state, It was found that $67.3 \%$ of the studied patients were married as compared to widows, neither single patient. The study results also supported by (Ahmed 2013). who showed that the highest percentage of the studied patients was married. This may be due to the higher level of daily activities, life strain on married than single one and that pressure is one of the greatest aggravating features for coronary artery disease.

Regarding to occupation the finding of the current study revealed that nearly one quarter $(24.0 \%)$ of patients were skilled this result disagree with (Ahmed 2013) who reported that the majority of patients were un employers. This is because moderate and strong physical activities usually linked with heart attack.

Concerning to the studied patients knowledge about symptoms of myocardial infarction, the current study show that two thirds of patients said that pressure or tightness in the chest is symptom of myocardial infarction. Slightly more than two fifths of patients explain that shortness of breath is the symptom of myocardial infarction. This agree with (Catrin et al., 2012) who reported from his study on Patients were recruited from cardiology departments of four Swedish hospitals, that people in general often know about chest pain as a symptom of an acute myocardial infarction (AMI), but commonly fail to recognize other warning signs. Patients usually interpret their symptoms as less serious and not warranting to call for an ambulance. Attitudes and opinions of other people also influence the decision time. as do psychological factors. Patients were recruited from cardiology departments of four Swedish hospitals

The current study shows that only less than one tenth from the total patients know the main causes of myocardial infarction. In opposite line with (Biala et al., 2018) who reported that the respondents considered atherosclerosis to be the main cause of myocardial infarction from the study about knowledge of risk factors of myocardial infarction in patients treated in the interventional cardiology unit of the regional specialist hospital in Biala Podlaska. .

Less than three quarters of patients in the current study said that smoking is the most risk factors for myocardial infarction. This result agree with (Sawicka et al., 2016) who study the awareness of risk factors for coronary heart disease in patients after myocardial infarction, in Poland, said that most of the respondents believe that smoking cigarettes raises the risk of developing cardiovascular diseases by five times. Less than one quarter of patients know that diabetes mellitus is the a risk factors for myocardial infarction.

Knowledge of acut myocardial infarction and primary percutaneous coronary intervention -symptoms remains to be substandard, especially knowledge of atypical-symptoms. Knowledge is essential to reduce delay-times, but it is not a panacea, since it is not sufficient alone to optimize pre hospital delay-times (Albarqounia et al., 2016).

Regarding to the family history the current study explain that more than half of patients had family history of coronary artery disease before the age of 55 years. This disagree with (Biala et al., 2018) who reported that all patients have coronary artery diseases, has family history also of these type of diseases.

Regarding to the studied patients knowledge about primary percutaneous coronary intervention (PPCI) . It was found that more than one tenths of patients know the definition of Primary percutaneous coronary intervention correctly. Also nobody of patients know that primary percutaneous coronary intervention is the appropriate reperfusion therapy. This is agree with (Mohammed et al., 2016) who designed nursing educational protocol on health promotion for patients undergoing coronary artery stent using convenience sample of sixty adult male and female patients aged from (18-65 years old) who are scheduled for non emergency coronary artery 
stent in catheterization lab at Assiut University Hospital, reported that, patients had no data and knowledge regarding cardiac catheterization.

This is due to lack of health education and instruction provided by nursing and medical staff member who did not offer them enough knowledge about their condition and this cause anxiety and distress from procedure.

Regarding to the patients reported practices It was found that more than three quarters of patients always eating food rich in fat and grease. Also the majority of patients always taking a lot of salt in their food. While study conducted by (Renata et al., 2017) who study the association between dietary factors and mortality from heart disease, stroke, and type 2 diabetes in the United States, explained that dietary factors were estimated to be associated with a substantial proportion of deaths from heart disease, stroke, and type 2 diabetes. This could be related to life long negative eating habits that patients must modify after incidence of the disease to maintain good health. These results should help identify priorities, guide public health planning, and inform strategies to alter dietary habits and improve health.

Slightly more than three fifths of them never practice any kind of exercise These results gone in the same line with (Tantaewy et al., 2013) who reported that around half of her studied patients (convenience sample of 50 adult patients admitted to coronary care unit of the Main University Hospital at Mansoura University), stated that most of patient did not perform any type of exercises. Moreover the present study shows that more than four fifths of patients were smokers in the present study.

Smoking is considered to be strong risk factor of myocardial infarction, premature atherosclerosis and sudden cardiac death. Smoking results in early STEMI especially in other-wise healthier patients. Cigarette smoking increases the risk for MI by multiple and complex mechanisms. (Vedika 2018)

\section{Conclusion}

Based on the results of the present study and research questions, it was concluded that more than half of the studied patients had myocardial infarction (MI) whom underwent primary percutaneous coronary intervention (PPCI) both sexes males and females had poor knowledge and reported practice regarding their diseases.

\section{Recommendations}

- Health education program is needed for patients with myocardial infarction to improve their knowledge and practice regarding their disease.
- Give booklet as handout for patients containing basic and important instructions for MI patients when leaving hospital.

\section{References}

1. Ahmed S., (2014): Impact of phase one cardiac rehabilitation on early complication of patients with st segment - elevation acute myocardial infarction in coronary care unit. master degree in critical care nursing, Faculty of Nursing; Assiut University.

2. Albarqouni L., Smenes K., Meinertz T., \& Schunkert H., (2016): Patients' knowledge about symptoms and adequate behavior during acute myocardial infarction and its impact on delay time Educ Couns;99(11):1845-1851

3. Alherbish A., Westerhout C., \& Afu Y., (2013): The forgotten lead: does aVR STdeviation add insight into the outcomes of STelevation myocardial infarction patients, Am Heart J. 166(2):333-9

4. Amsterdam E., Wenger N., Brindis R., Casey D., Ganiats T., Holmes D., Jaffe A., Jneid H., Kelly R., Kontos M., Levine G., Liebson P., Mukherjee D., Peterson E., Sabatine M., Smalling R., \& Zieman S., (2014): AHA/ACC Guideline for the Management of Patients With Non-ST-Elevation Acute Coronary Syndromes: Circulation J. 130(4): 344- 426.

5. Biała P., Ślifirczyk A., Michalczuk T., Piszcz P., Kowalenko M., Kierczuk E., Zalewski R., 2B-C \& Krajewska - Kułak E., (2018): Knowledge of risk factors of myocardial infarction in patients, Prog Health Sci, 8(1): 97.

6. Catrin H., Margareta L., Judy A., Johan H., Jan-Erik K., Leif S., Marie T., Crister Z., Lisa W., (2012): Knowledge about Acute Myocardial Infarction (AMI) and attitudes to medical care seeking-A comparison between, patients and the general public, Open Journal of Nursing, , 2, 372-378

7. Crawford M., Boyle M., Murgo M., \& Adamson H., (2009): The effect of chronic pain on health related quality of life amongst intensive care survivors. Aust Crit Care; 17: 104 $-113$

8. Gary H., \& Gibbons, M., (2015): "What Are the Signs and Symptoms of Coronary Heart Disease, National heart ,lung and blood institute 67(5): 802-807

9. Garza A., Gratton M., \& Salomone J., (2009): Improved Patient Survival Using a Modified Resuscitation Protocol for Out-of-Hospital 119(19):2597-605. 
10. Global Burden of Disease (2016): "Global, regional, and national incidence, prevalence, and years lived with disability for 310 diseases and injuries, 1990-2015: a systematic analysis ". Lancet. 388 (10053): 1545-1602.

11. Harold J., Bass T., \& Bashore T., (2013): Coronary Artery Interventional Procedures, Clinical Competence. Circulation. 128 (4): 43672.

12. Maziar Z., \& Eric Y., (2016): Myocardial Infarction Overview Presentation D Dx Workup Treatment Medication. 388( 10053) :1459-1544.

13. Mehta P., Wei J., \& Wenger N., (2014): "Ischemic heart disease in women: A focus on risk factors" Trends in Cardiovascular Medicine. 25 (2): 140-151.

14. Mohammed G., Mohammed Z., \& Al-Araby H., (2016): Impact Of De-signed Nursing Educational Protocol On Health Promotion For Pa-tients Undergoing Coronary Artery Stent Outcome IOSR Journal of Nursing and Health Science 5(2): 54-63.

15. Renata M., Jose L., Peñalvo, Frederick ., Fumiaki I., Colin D., \& Dariush M., (2017): Association Between Dietary Factors and Mortality From Heart Disease, Stroke, and Type 2 Diabetes in the United States JAMA. 317(9):912-924.

16. Sawicka K., Grządka A., Luczyk R., Wawryniuk A., Prasal M., Luczyk M., \& Daniluk J., (2016): Knowledge of risk factors of myocardial infarction in patients. Health Sport; 6 (12):795-816.

17. Tantaewy N., Amany M, Shereif W., \& Ismail S., (2013): Effect of Cardiac Rehabilitation Program on Lifestyle Pattern of Patients with Myocardial Infraction Journal of Biology, Agriculture and Healthcare 3(8): 2225-093

18. Vedika R., Neelima S., \& Roshan K., (2018): Risk Factors for Acute Myocardial Infarction, Eurasian Journal of Medicine and Investigation Available online at www.ejmi.or

19. Wijesinghe N., Christopher N., Cherian S., Spencer H., Hugh Mc. A., \& Gerard D., (2018): Complications of Primary Angioplasty in Myocardial Infarction (PAMI) in a Real-World Cardiac Catheterisation Laboratory: 8-Year Experience Heart and lung J;17: (3): 175

20. World health rankings (2017): live longer live better Egypt : coronary heart disease

21. Wright J., Williamson J., \& Whelton P., (2015): The SPRINT Research Group. A randomized trial of intensive versus standard blood-pressure control. N Engl J Med; 373: 2103-2116. 